\title{
Miniature On-Board Angle of Attack Measurement System for Hypersonic Facilities
}

\author{
Bradley L. Crawford ${ }^{*}$ and Matthew N. Rhode ${ }^{\dagger}$ \\ NASA Langley Research Center Hampton, Virginia 23681
}

\begin{abstract}
I. Abstract:
The most prevalent method of establishing model angle of attack (AoA) in hypersonic wind tunnel facilities is using an encoder in the model support system then calculating sting/balance deflections based on balance output. This method has been shown to be less accurate than on-board methods in subsonic and transonic facilities $^{i}$ and preliminary indications, as compared to optical methods, show large discrepancies in a hypersonic facility as well. With improvements in Micro-ElectroMechanical Systems (MEMS) accelerometer technology more accurate onboard AoA measurement systems are now available for the small models usually found in hypersonic research facilities.
\end{abstract}

\section{Introduction:}

The AoA accuracy requirement for hypersonic facilities is currently $0.1^{\circ}$. MEMS accelerometers are capable of this accuracy under lab conditions but are susceptible to environmental conditions such as temperature and vibration. Some unique techniques have been employed to counter these situations that will allow an on-board accelerometer instrumentation system to meet this requirement. Judiciously selecting and packaging the instruments in pairs then reading them differentially creates a tremendous benefit. Vibration isolation can limit the dynamic excitation levels keeping it within the accelerometer's range. These concerns along with lab and tunnel data will be discussed in this paper.

A test of a newly designed MEMS AoA accelerometer instrumentation system was conducted in

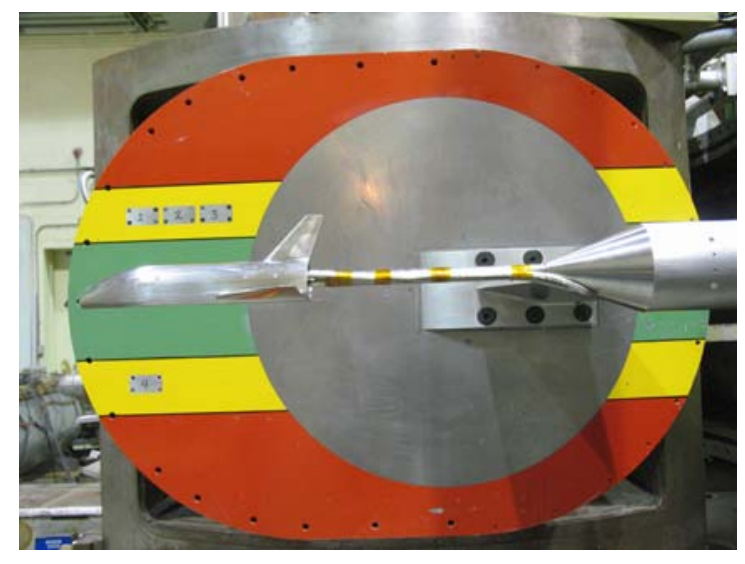

Figure 1: Generic orbiter model mounted to tunnel injection system the NASA Langley Research Center 31-Inch Mach 10 Tunnel using a generic orbiter-type configuration as the test article (Figure 1). The 31-Inch Mach 10 Tunnel is a blowdown-to-vacuum facility utilizing dry air as the test gas. The preheated air flows through an inline filter before expanding through the three-dimensional contoured nozzle and into the test section. A sidewallmounted injection system is used to place the model into the test section following establishment of the flow and to remove the model prior to stopping the flow.

The MEMS accelerometer instrumentation was installed on the front of the balance-to-model adaptor inside of the model. An air gap on three sides of the adaptor, plus the proximity of the accelerometer to the water-cooled balance should reduce the thermal effects on the instrumentation. The sequence of angle of attack points was varied at random to guard against bias effects.

\footnotetext{
* Electronics Engineer, Aeronautics Systems Engineering Branch, M/S 238, AIAA Senior Member

${ }^{\dagger}$ Aerospace Engineer, Aerothermodynamics Branch, Mail Stop 408A, AIAA Senior Member
} 
Hypersonic wind tunnel facilities are in need of new techniques for measuring AoA and the onboard method mentioned above is capable of meeting the requirements once the challenges have been overcome. In this paper the techniques are discussed in detail, test procedures will be discussed, lab and tunnel test results will be compared and presented and a discussion of follow-on work will conclude.

\section{MINI AoA:}

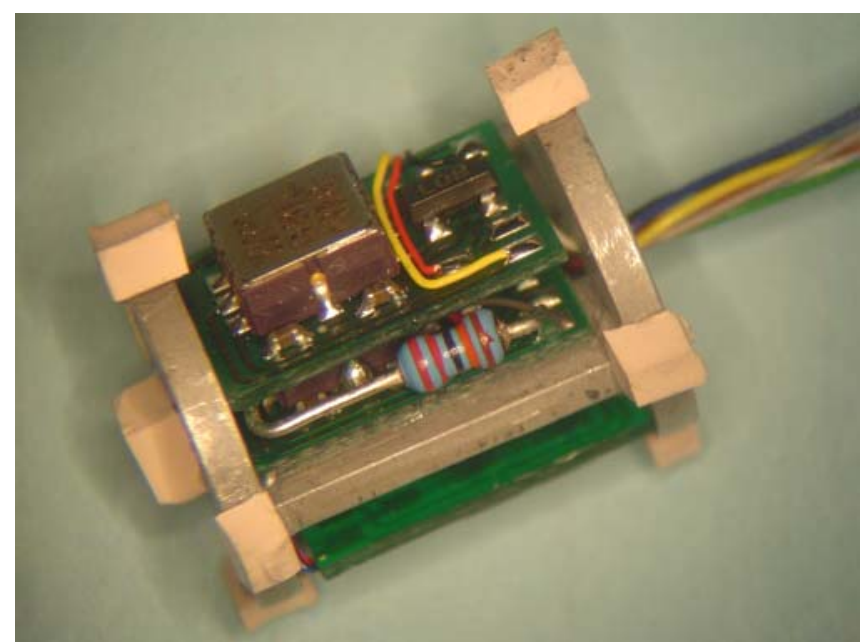

Figure 2: MINI AoA with 10g and $3 \mathrm{~g}$ accelerometers

MEMS accelerometers have advanced to a state where they now are being considered for use as angle of attack sensors in wind tunnel models. They are desirable because of reduced cost, size and weight. The miniature angle of attack sensor (MINI AoA), pictured in Figure 2 without enclosure, shows a configuration of accelerometers with two $3 \mathrm{~g}^{\mathrm{iii}}$ accelerometers and two $10 \mathrm{~g}^{\mathrm{iv}}$ accelerometers. This design can fit into a $5 / 8$ " dia. x $7 / 8$ " long canister as opposed to the Langley standard AoA package which is 1.12 " dia. $x 1.37$ " long.

The MINI AoA has been configured with two sets of accelerometers for phase one testing to ensure that environmental characteristics are captured. The $3 \mathrm{~g}$ accelerometers are the sensors of choice for accuracy reasons but there is a lack of information on model vibration characteristics in hypersonic

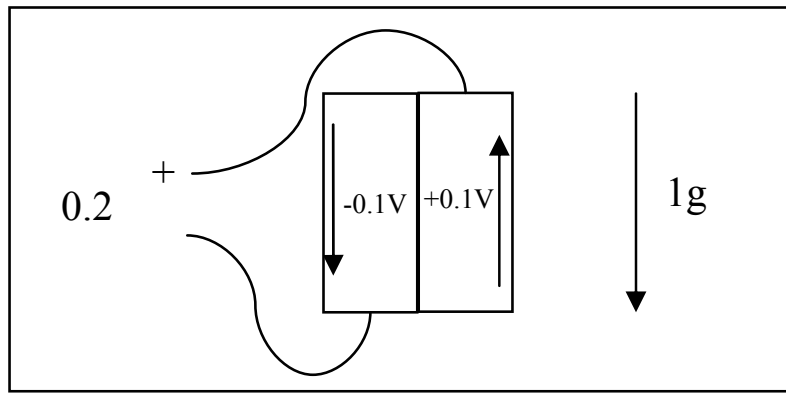

Figure 3: Sensor differential reading facilities. The possibility existed that the $3 \mathrm{~g}$ accelerometers could be saturated so the $10 \mathrm{~g}$ accelerometers were included in the package to ensure that the vibration characteristics were captured.

Sensors were paired in the instrumentation housing and read differentially. Pairing the sensors has several benefits. By reading the high output sensor with respect to the low output sensor the $\sim 2.5 \mathrm{~V}$ bias is all but eliminated. Using Figure 3 as an example, if the sensors were read individually the $+1 \mathrm{~g}$ sensor would read $2.6 \mathrm{~V}(2.5 \mathrm{~V}$ bias $+0.1 \mathrm{~V} / \mathrm{g}$ output) the $-1 \mathrm{~g}$ sensor would read $2.4 \mathrm{~V}(2.5 \mathrm{~V}$ bias $-0.1 \mathrm{~V} / \mathrm{g}$ output $)$. When the $+1 \mathrm{~g}$ sensor is read with respect to the $-1 \mathrm{~g}$ sensor the resulting voltage is $0.2 \mathrm{~V}$. Not doing this would require the tunnel data system to range the channel to a relatively high range (at least $2.6 \mathrm{~V}$ ) but only have a $0.2 \mathrm{~V}$ change in output. Reading them differentially allows the data system to be set to a lower range $( \pm 0.2 \mathrm{~V}$ min.) generating much higher resolution for the reading. 
As seen in the above example, pairing sensors doubles the sensitivity but another benefit is that the noise only rises by a factor of $\sqrt{2}$ giving a better signal to noise ratio (SNR). For example, if a single sensor had a $1 \mathrm{~V}$ output in a $1 \mathrm{~g}$ field and a noise of $0.1 \mathrm{~V}$ the $\mathrm{SNR}$ would be $1 \mathrm{~V} / 0.1 \mathrm{~V}=10$. By reading them differentially the output would be $2 \mathrm{~V}$ and the noise would be $0.14 \mathrm{~V}$ so the SNR would be $2 \mathrm{~V} / 0.14 \mathrm{~V}$ $=14.3$. Another benefit of the pairings is the ability to match sensors with like temperature characteristics. By doing this, the temperature characteristics can be drastically reduced. The upper left chart of Figure 4 shows the bias shift with temperature of sensor \#1 and the upper right chart shows the bias shift with temperature of sensor \#2. When the two sensors are aligned with opposite polarity and read differentially the result is a drastically reduced bias temperature effect as shown in the bottom chart.
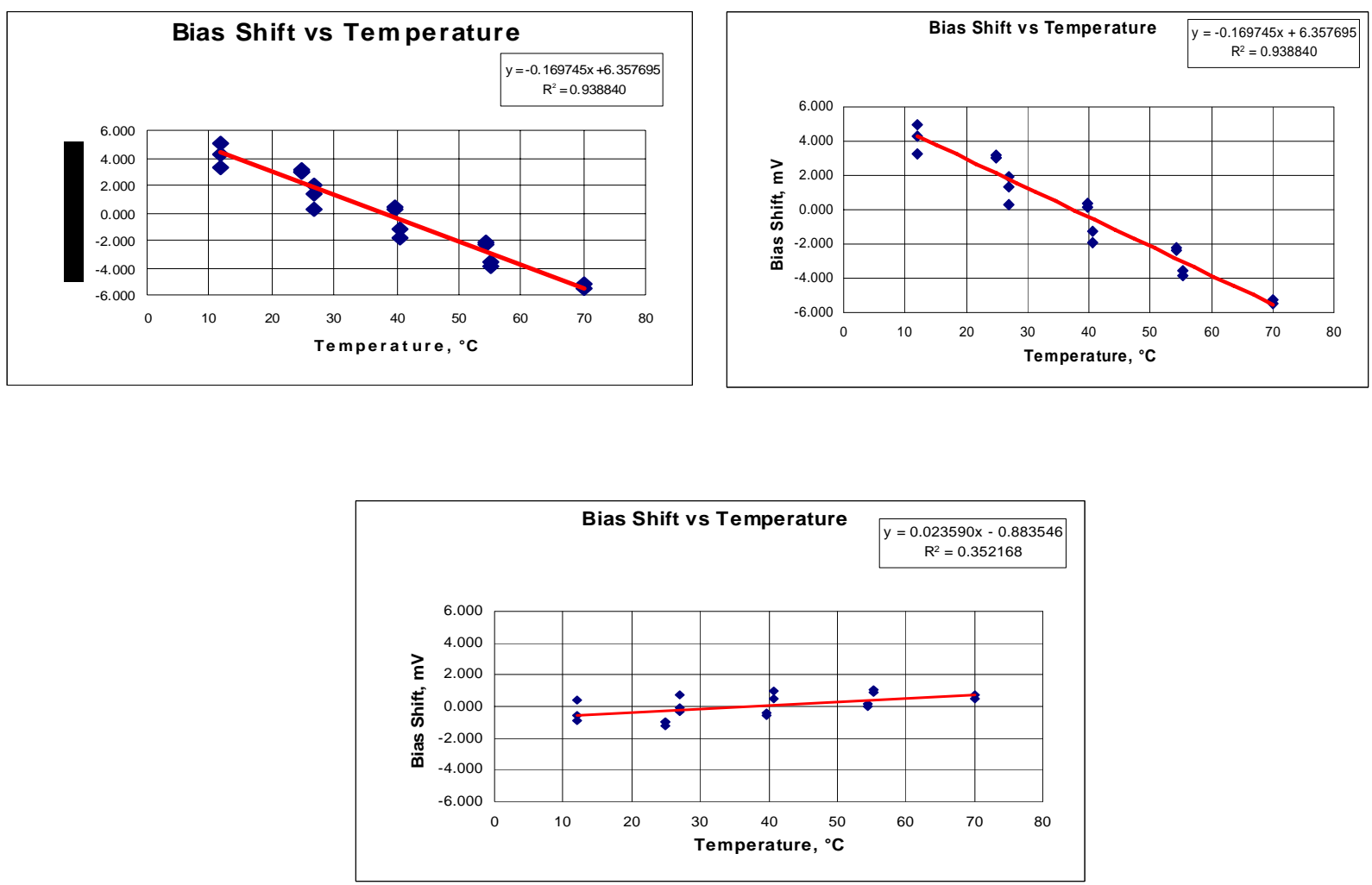

Figure 4: Matching MEMS temperature characteristics for better performance

If the characteristics could be exactly matched then the bias shift would be totally eliminated by this method. Of course this is impossible so an analog method of reducing this bias is to shunt the output of the steepest sloped sensor. Finally, a mathematical model can be applied to any remaining temperature effect and reduce it even further.

In this case a $2^{\text {nd }}$ order model was used as follows:

$\mathrm{B}=\mathrm{B}_{0}+\mathrm{B}_{1} * \mathrm{~T}+\mathrm{B}_{2} * \mathrm{~T}^{\wedge} 2$

Where $\mathrm{B}$ is the temperature compensated bias, $\mathrm{B}_{0}, \mathrm{~B}_{1}$, and $\mathrm{B}_{2}$ are the bias temperature coefficients and $\mathrm{T}$ is the current temperature. The sensitivity and offset can be corrected similarly with the coefficients $\mathrm{S}, \mathrm{S}_{0}$, $\mathrm{S}_{1}, \mathrm{~S}_{2}$ and $\mathrm{O}, \mathrm{O}_{0}, \mathrm{O}_{1}, \mathrm{O}_{2}$ respectively. Once the sensor is installed and ready to go a final in-situ calibration needs to be performed. The second order temperature effects equation establishes the fixed 
curvature for the sensor but the $\mathrm{B}_{0}, \mathrm{~S}_{0}$ and $\mathrm{O}_{0}$ coefficients need to be adjusted to establish the position of the curve. This is accomplished according to equation 2.

$\mathrm{B}_{0 \mathrm{a}}=\mathrm{B}_{\mathrm{c}}-\mathrm{B}_{1} * \mathrm{~T}_{\mathrm{c}}-\mathrm{B}_{2} * \mathrm{~T}_{\mathrm{c}}{ }^{\wedge}$

Where $\mathrm{B}_{0 \mathrm{a}}$ becomes the adjusted zeroth order bias coefficient that is substituted for the $\mathrm{B}_{0}$ coefficient in eq. $1, B_{c}$ is the bias established from the in-situ calibration and $T_{c}$ is the temperature during the in-situ calibration.

This can best be illustrated using the $2^{\text {nd }}$ order offset equation as an example. The offset temperature coefficients $\mathrm{O}_{0}, \mathrm{O}_{1}$ and $\mathrm{O}_{2}$ are established in the lab. When the sensor is placed in the model there is a mechanical misalignment between where the sensor was aligned relative to gravity during the lab calibration and the way it is aligned in the model at the tunnel. When the in-situ calibration is performed in the tunnel this new offset is established at some new temperature. Both the new offset and in-situ calibration temperature are taken into consideration when adjusting the $\mathrm{O}_{0}$ coefficient as per equation 2.

When used in more favorable conditions such as the lab, the MINI AoA measurements can be quite good as seen in the comparison to a precision indexing head capable of 1 arc second of accuracy. This indexing head is used as a reference standard for the MINI AoA and the data shows that it can achieve accuracies of better than $0.005^{\circ}$ (Figure 5).
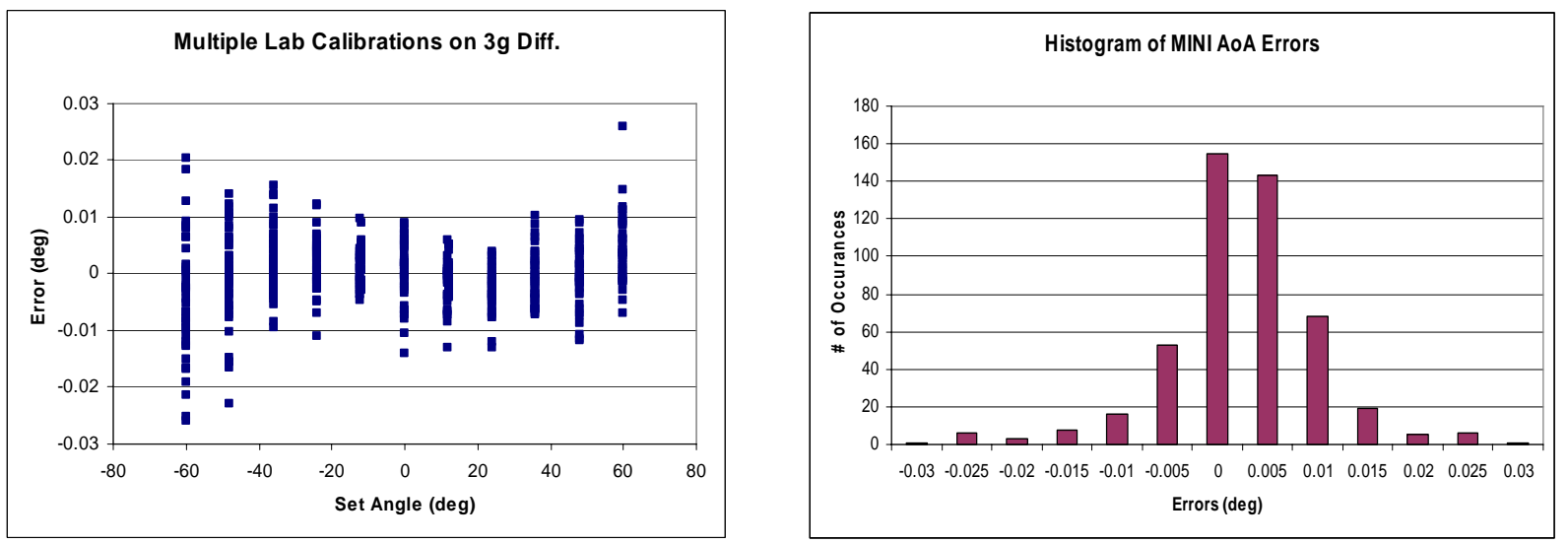

Figure 5: Repeated accuracy of the MINI AoA during lab testing

In this example, $60 \%$ of the points lie within $0.005^{\circ}$ and the $2 \sigma$ value is $0.025^{\circ}$ over a range of $\pm 60^{\circ}$. This is a very wide range for most tunnels during a test but is often used during lab calibrations because it produces a better estimate of the bias. If you consider a more realistic range of $\pm 24^{\circ}$ then the $2 \sigma$ value is about $.009^{\circ}$.

\section{The Facility:}

The 31-Inch Mach 10 Tunnel $^{\mathrm{v}}$ is a blowdown wind tunnel that uses dry air as the test gas. Air is transferred from a high-pressure bottlefield to a reservoir where the air is heated to a maximum temperature of approximately $1820 \mathrm{R}$ by a $12.5-\mathrm{MW}$ electrical resistance heater. An inline, sintered metal filter is located between the heater and the settling chamber to catch particles larger than $5 \mu \mathrm{m}$ in size. The settling chamber contains a number of internal screens to aid flow mixing and reduce turbulence in the air stream. From the settling chamber, the air expands through a water-cooled, square 
cross section, contoured nozzle and into a test section $31 \times 31$ inches in cross section and approximately 34.5 inches in usable length. An adjustable second minimum is located downstream of the test section to improve pressure recovery. The flow exhausts through an aftercooler and into a combination of 41 and 60 -foot-diameter vacuum spheres that are pumped down by mechanical vacuum pumps and a three-stage steam ejector. Maximum run time is approximately 2 minutes depending on the operating conditions and the available vacuum capacity.

Models are supported on a hydraulically operated, sidewall-mounted, variable-speed injection system. The injection system is used to place the model into the flow from a sheltered position following flow establishment and to remove the test article prior to flow stoppage, in both instances preventing strong dynamic loads on the model. A flush injection plate fills the opening in the side of the test section wall when the model is injected into the tunnel. A computer controlled arc sector located on the injection plate provides pitch angles between \pm 45 degrees, and yaw angle can be adjusted manually between runs to angles of up to \pm 5 degrees. Models are mounted to the injection system using a variety of strut and sting hardware.

The facility has been calibrated at stagnation pressures from 250 to 1300 psia and stagnation temperatures from 1720 to $1820 \mathrm{R}$. Over these pressure and temperature ranges, the freestream Mach number varies from 9.15 to 9.85 , with freestream unit Reynolds numbers from 0.4 to $2.0 \times 10^{6}$ per foot. The inviscid core flow size ranges from \pm 5 to \pm 7 inches, with variations in pitot pressure of less than \pm 2 percent.

The 31-Inch Mach 10 Tunnel has a stand-alone data system with hardware and software identical to other facilities in the Langley Aerothermodynamics Laboratory (LAL). The core of the system is a 256-channel, amplifier-per-channel data acquisition system manufactured by the NEFF Instrument Corporation. Each channel contains programmable gains and filters, with signals multiplexed to a 16-bit analog-to-digital converter. A signal conditioner provides up to \pm 10 volts excitation to various instruments. A typical sampling rate is 50 samples per second for each channel, although much higher rates are possible. Presently, 512 channels of pressure measurements are available via electronically scanned pressure (ESP) sensors using a Pressure Systems, Inc. System 8400. ESP modules containing up to 64 sensors and of various pressure ranges are on hand for use in the facility, and other pressure instrumentation is available for use in certain applications. Two PC-type computers are used to control the data system; one is dedicated to data acquisition while the other is used for data reduction. Data are typically available only minutes following a run.

The AOA sensor evaluation was conducted using the LAL Check Standard Model (CSM). The CSM was originally built to assess long-term data repeatability in LAL facilities using conventional data quality assurance methods. A generic orbiter-type configuration with a length of 8.5 inches and a wingspan of 4.7 inches, the model was fabricated from one piece of stainless steel and employs a number of balance adapters for use with several Langley force and moment balances. The adapters attach to the upper inside surface of the model, providing a substantial air gap between the adapter and the windward surface, lessening heat conduction to the balance during an operational run. For this test, a new balance adapter was built with attachment points for mounting the AoA sensor housing directly to the adapter and in close proximity to the water-cooled force balance, limiting the heat flux to the AoA sensors. The model was supported on a straight sting through the base of the model, and force and moment measurements were obtained using the 0.5625 -inch-diameter, water-cooled, six-component balance SS17B.

\section{The Test:}

This test is the culmination of the first development phase for the miniature AoA and is being conducted in NASA Langley Research Center's 31-Inch Mach 10 Tunnel. This facility is representative of the hypersonic facilities that the MINI AoA is being developed for. 


\section{A. Setup:}

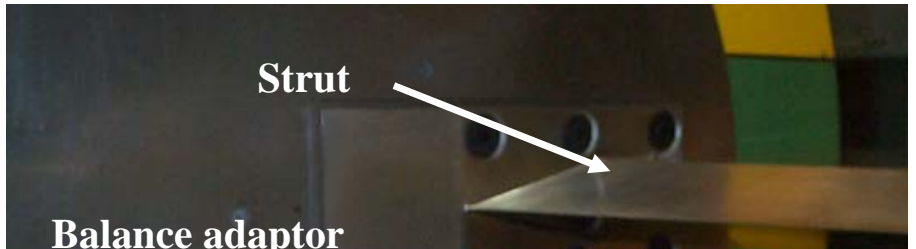

A generic orbiter type model mounted on a strut, sting and balance support assembly was chosen as the vehicle for this test. It was run as a designed experiment of two factors, pressure and angle of attack. Pressure and AoA were randomly set with pressure randomized for each run and the AoA randomized within a run. Pressures chosen for this test were 350 and 1300 psi and the AoA angles were $-5^{\circ}, 5^{\circ}$ and $15^{\circ}$ with the endpoints repeated during each run for a total of 5 data points per run. This repeated endpoint test strategy allowed for testing of curvature. Pitch pause polars were run with pitch settings set in random

Figure 6: MINI AoA installation before model assembly order and a $8 \mathrm{~s}$ dwell time at each pitch angle to allow for sensor lag. The MINI AoA was mounted on the front end of the balance adaptor as shown in Figure 6.

\section{B. Objectives:}

For this test the primary objectives were to characterize the environmental parameters of temperature and vibration, and secondary objectives were to perform preliminary accuracy checks on the MINI AoA. Two environmental effects are of most concern, temperature and vibration. Accuracy was assessed by direct comparison and by statistical evaluation of the raw data comprising each averaged data point.

The maximum operating temperature for the chosen sensors is $125^{\circ} \mathrm{C}$ and the excitation level for the $3 \mathrm{~g}$ sensors is $\pm 1.7 \mathrm{~g}$ and the $10 \mathrm{~g}$ sensor is $\pm 10 \mathrm{~g}$. To check the heat build-up during runs an integrated circuit (IC) temperature transducer was located in the sensor container and the temperature was monitored from the start of each run until well after the runs were complete. The transducer output is $1 \mu \mathrm{A} / \mathrm{K}$ but to acquire the data, a voltage is measured across a $1 \mathrm{~K} \Omega$ resister making the sensor sensitivity $1 \mathrm{mV} / \mathrm{K}$.

To capture the vibration characteristics of the support assembly, a Fast Fourier Transform (FFT) analyzer was connected to the unused second axis of the $10 \mathrm{~g}$ accelerometer oriented in the model normal direction. The analyzer performed the transform then output the results to a notebook computer for storage. The FFT's were performed at intervals during the runs that depended on the speed of the analyzer (3 to 4 analysis per run).

To assess the accuracy of the MINI AoA, comparisons were to be made to the tunnel's standard encoder AoA estimate that includes the adjustments for sting deflections and flow angularity and to a remote optical system. Unfortunately the optical system encountered a problem with the calibration that was not discovered until the data was being post-processed. Having only the tunnel encoder's estimate of AoA to verify the on board AoA was not very satisfactory especially given that the encoder estimate is what this test is meant to replace. 


\section{In-situ Calibration:}

The in-situ calibration was performed using a LaRC custom-built angle measurement system (AMS) as a transfer standard. AMS packages have a stated $2 \sigma$ pitch accuracy after calibration of $0.002^{\circ}$

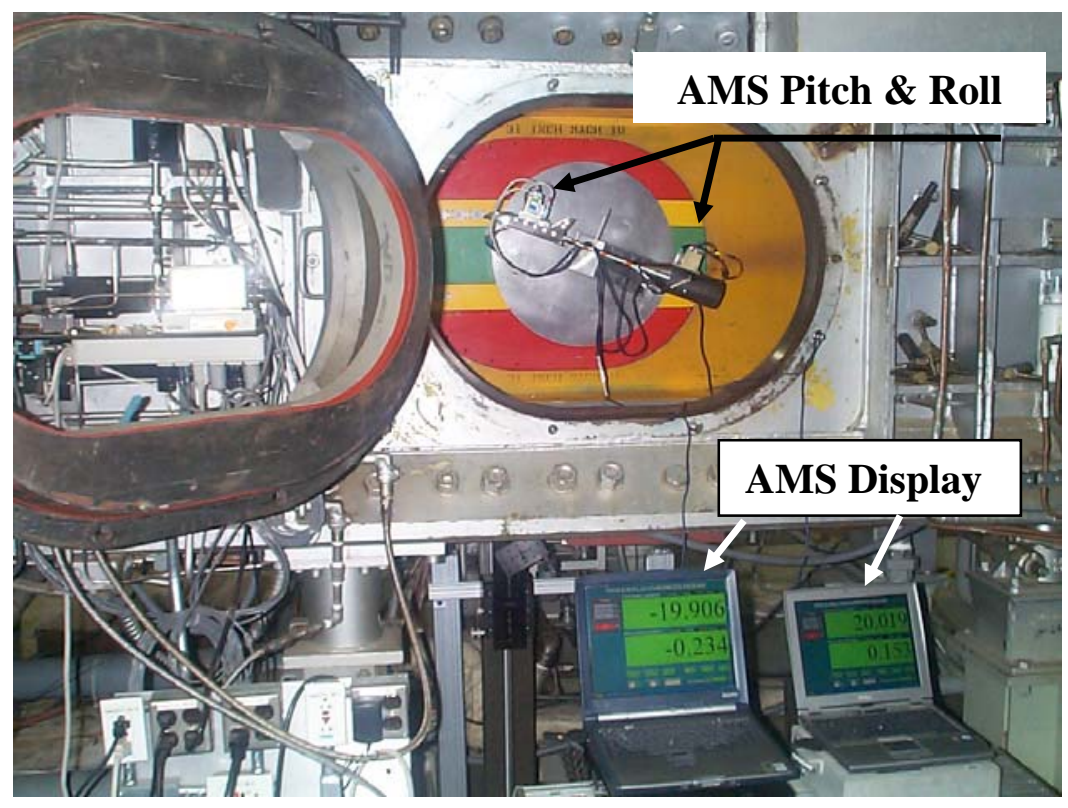

Figure 7: In-situ calibration using AMS between $\pm 30^{\circ}$. Finley's paper $^{\mathrm{vi}}$ discusses the AMS predecessor in detail. The tunnel AoA encoder and the MINI AoA were calibrated against the AMS (Figure 7) simultaneously by mounting the pitch \& roll sensor package on top of the model (even though the model was locked in the roll axis). A wind off polar was then performed over an angle range of $-10^{\circ}$ to $20^{\circ}$ in random order of $5^{\circ}$ increments. The output from the encoder, MINI AoA and the AMS were monitored and individual regressions were performed on the sensors against the AMS reference.

The regression residuals in Figure 8 show the wind off accuracy for the three estimates of AoA in this test, the $10 \mathrm{~g}$ and $3 \mathrm{~g}$ differential readings and two separate readings from the tunnel encoder. This regression generated the $\mathrm{B}_{\mathrm{c}}$, $\mathrm{S}_{\mathrm{c}}$, and $\mathrm{O}_{\mathrm{c}}$ terms for use in eq. 2 (for both the $10 \mathrm{~g}$ and $3 \mathrm{~g}$ accelerometers) to calculate the new zeroth

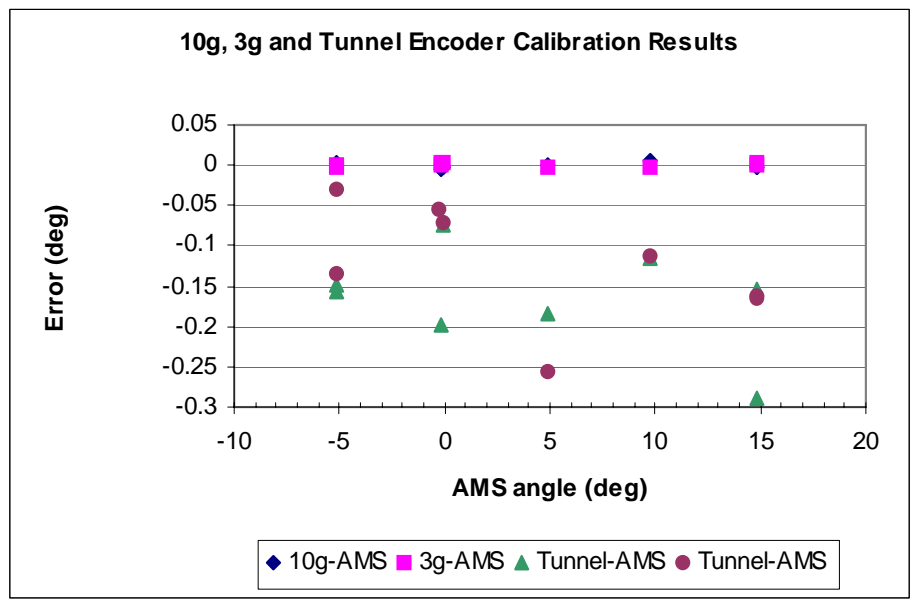

Figure 8: Residual errors for MINI AoA (3g and 10g accelerometers) and the tunnel encoder as compared to the order coefficients. It can be seen in Figure 8 that the $3 \mathrm{~g}$ and $10 \mathrm{~g}$ accelerometers were well behaved while the tunnel encoder estimate has an error of nearly $0.3^{\circ}$.

The accelerometers have a large margin for wind-on induced errors before they reach the $0.1^{\circ}$ error budget limit. This margin should be enough to handle temperature and vibration effects but may not cover "sting whip"vii induced errors (errors in AoA generated by centrifugal acceleration as the model "whips" on the end of the sting). At this stage of the MINI AoA development sting whip is acknowledged as a potential error source but is not being addressed.

\section{Results:}

The hardest part of an experiment like this is the lack of an absolute reference to measure the readings against. In the calibration portion of this paper the AMS was used during wind-off runs as a standard for calibration and to verify instrument accuracies. The AMS has to be 


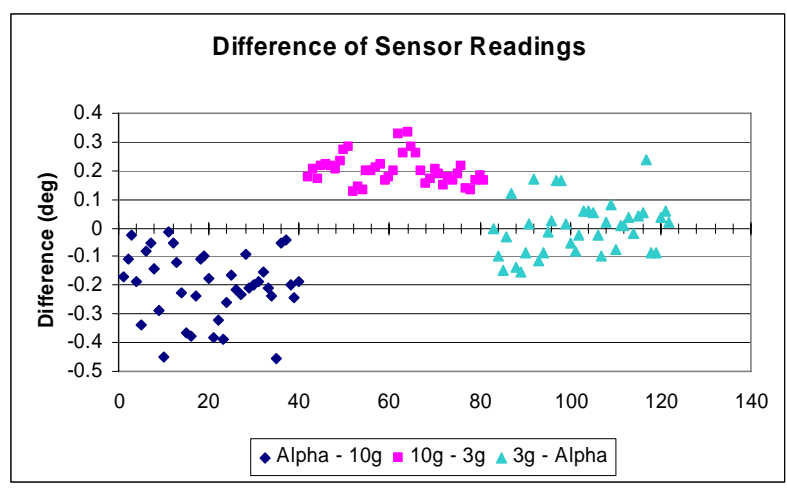

Figure 9: Differences between the sensor readings removed from the model during wind-on runs to prevent damage to the instrument, model or tunnel. That, combined with the problems encountered with the optical system, leaves the test without an independent AoA estimate to use as a reference. As a work around to this problem a round-robin approach can be employed whereby each of the three AoA estimates is subtracted from the other two estimates. When the three differences are plotted it is hoped that one of the readings will stand out differently than the others so inferences can be made. Figure 9 shows these differences plotted verses an arbitrary abscissa to separate them for clarity. Notice that both sets of data involving the $10 \mathrm{~g}$ accelerometer (blue and pink on the graph) are displaced from the axis. This implies that the $10 \mathrm{~g}$ accelerometer has some offset/bias in it. Also notice that the difference between the $3 \mathrm{~g}$ and the $10 \mathrm{~g}$ accelerometers has a $2 \sigma$ value of $0.1^{\circ}$ where as the tunnel alpha minus the $10 \mathrm{~g}$ accelerometer has a $2 \sigma$ of $0.23^{\circ}$ and the $3 \mathrm{~g}$ accelerometer minus alpha has $2 \sigma$ of

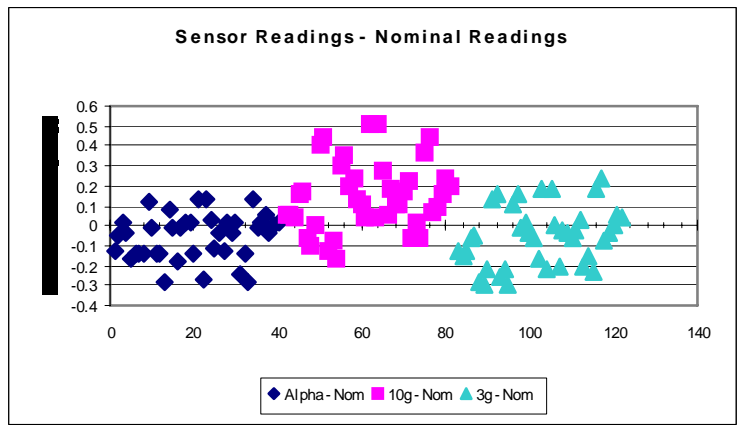

Figure 10: Instrument readings minus nominal set point $0.18^{\circ}$. This tells us that there is better agreement between the two accelerometers than between either of the accelerometers and the alpha encoder.

Another way is to use the nominal AoA set point then subtract it from each of the estimates (Figure 10). This at least gives a common independent reference point to assess the techniques. Notice here that the bias in the alpha data is drastically reduced while the $10 \mathrm{~g}$ accelerometer data is still offset. This confirms the notion from the previous data that the $10 \mathrm{~g}$ accelerometer had a bias

The world of wind tunnel measurements offers another means of comparison. Many times the desired wind tunnel measurement is a static one but the measurement is being made in a dynamic environment. For this reason data is typically taken over some period of time then averaged. In this test the data rate was $30 \mathrm{~Hz}$ and

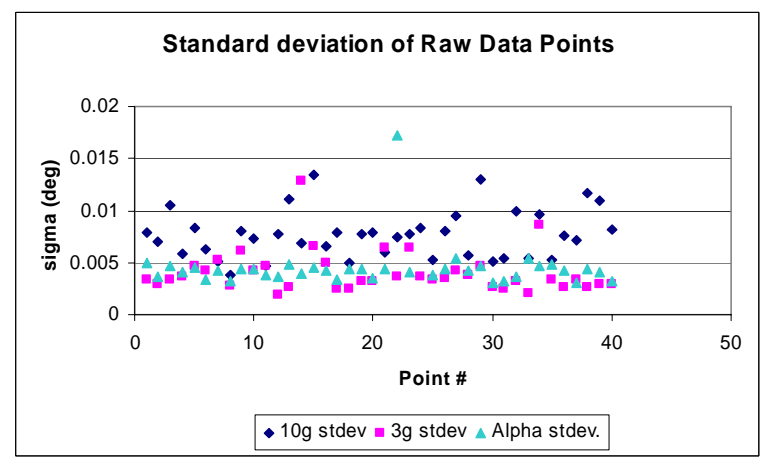

Figure 11: Standard deviations of raw data the data was averaged over a $4 \mathrm{~s}$ period providing 120 raw points per averaged data point. This raw data can be analyzed to establish the noise or scatter in each reading by taking the standard deviation of the raw values. This data (Figure 11) shows that the $10 \mathrm{~g}$ sensor is the noisiest with $2 \sigma$ $=0.015^{\circ}$, the $3 \mathrm{~g}$ sensor has $2 \sigma=.008^{\circ}$ and the tunnel's encoder $2 \sigma=0.009^{\circ}$. There is a problem with this method that needs to be pointed out here. That is, the encoder noise is not a true reflection of the noise level of the tunnel alpha 
reading. Sting deflection calculations were not available for the raw data so any noise that would have been contributed from this calculation would not have shown up in the encoder noise. The noise level in these readings would likely to go up when that noise source is included. Conversely, some of this "noise" content in the accelerometer data is from actual model movement that cannot be separated from the noise. This noise level would likely to drop if separation could be achieved.

The environmental affects of temperature and frequency content were also monitored

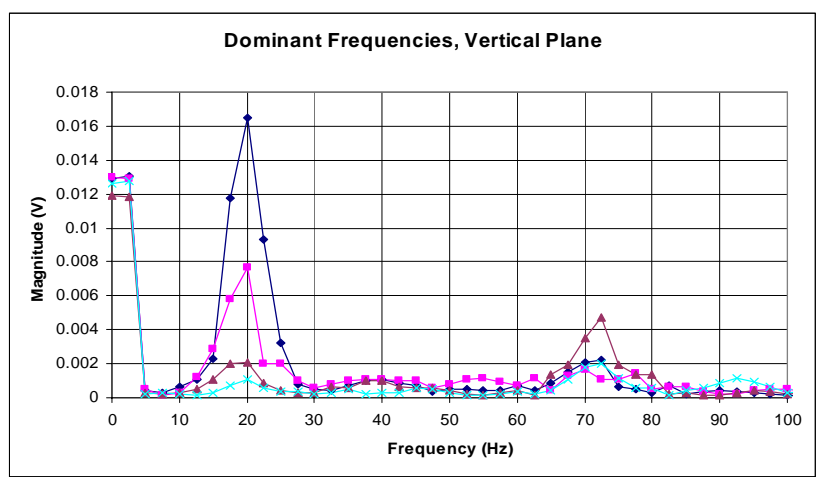

Figure 12: Sample of dominant frequencies during test during the testing. To establish the frequency content in this test configuration a Fast Fourier Transform (FFT) analyzer was used. It was started with a trigger from the model injection system and allowed to process data continuously throughout the roughly 80 second runs. After each analysis the results were transferred to a computer for storage. For this particular model and set up there were no visible signs of vibration during wind-on conditions but Figure 12 shows that there were predominant resonant frequencies around $20 \mathrm{~Hz}$ and $70 \mathrm{~Hz}$.

The MINI AoA temperature prior to

testing started at a minimum of just over $25^{\circ} \mathrm{C}$ and rose to just less than $28^{\circ} \mathrm{C}$ at the end of data taking then to a maximum of $33^{\circ} \mathrm{C}$ by the time the model was removed from the flow. However, after the model was removed from the flow and the flow was stopped, temperature rose by as much as $34^{\circ} \mathrm{C}$ to $61^{\circ} \mathrm{C}$. Even this is well within the maximum operating temperature of $125^{\circ} \mathrm{C}$. There was a relatively little temperature change during the data taking time period, generally
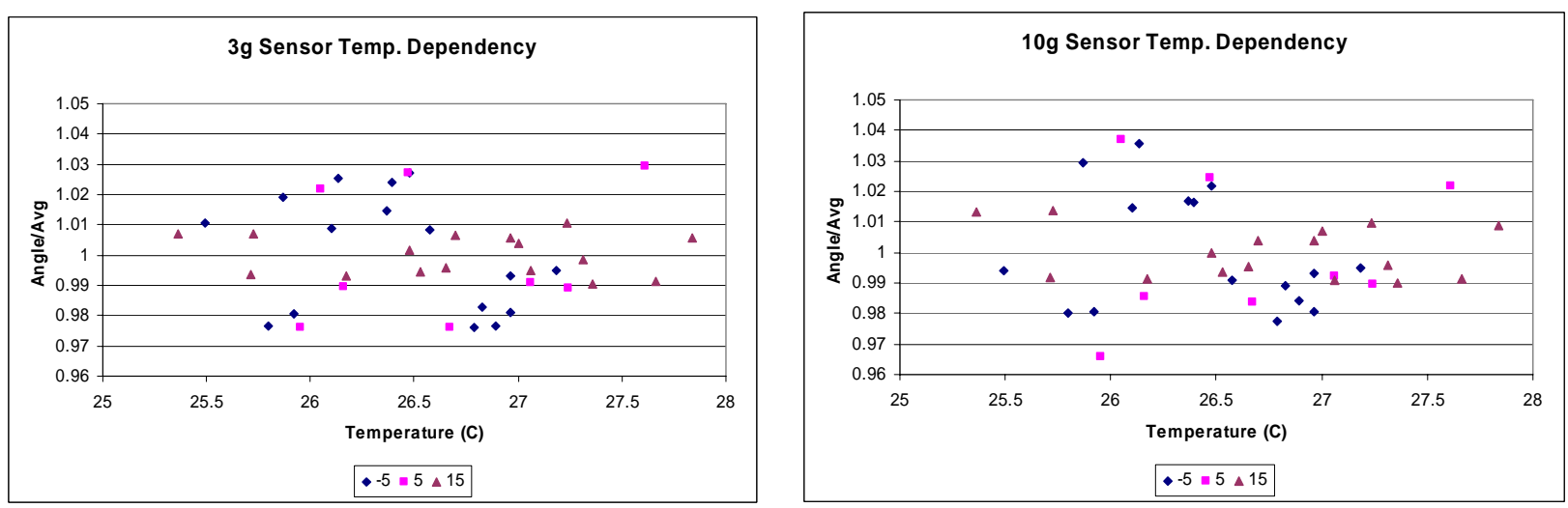

Figure 13: Temperature dependence of $3 \mathrm{~g}$ and $10 \mathrm{~g}$ accelerometers

about $2.5^{\circ} \mathrm{C}$. Looking at this data no temperature dependence can be seen (Figure 13) . 


\section{Conclusions:}

This test concluded the first phase of the development effort for a miniature onboard AoA sensor to be used in hypersonic facilities and the primary objectives of characterizing the environmental issues were met and preliminary indications of assessing AoA accuracy were promising.

The test showed that the environmental concerns of vibration and temperature are tolerable. Vibration resonances are of high enough frequency that filtering can be developed for good smoothing characteristics without initiating a large lag in the data. Temperatures were well within survivability limits of the sensors but additional work needs to be done to determine if temperature affects the AoA readings.

Due to the problems encountered with the optical technique the test was left without independent observation to use as an absolute angle reference. This makes it difficult to conclude the accuracy of the MIMI AoA sensor. Considering the noise issues discussed above and the calibration results the $3 \mathrm{~g}$ accelerometer probably gives a better indication of the AoA reading than the tunnel encoder based system. There are improvements that can be made on the MINI AoA system in the form of signal conditioning, and verification using an established independent reference system, still need to be performed to make this a usable everyday sensor.

\section{Acknowledgements:}

I would like to thank the Aerothermodynamics Branch, Research and Technology Directorate for supporting this work, the many people involved in the operation of the facility, Toby Comeaux for his technical support during development and testing of this sensor and Matt Rhode the test engineer and co-author. I would also like to show my appreciation to Dr. Peter Parker for his support in developing the test matrix and analysis of the data.

\footnotetext{
${ }^{\mathrm{i}}$ Crawford, Bradley L., Finley, Tom D.; "Results From a Sting Whip Correction Verification Test at the Langley 16-Foot Transonic Tunnel", 40th AIAA Aerospace Sciences Meeting and Exhibit, Reno, Nevada, AIAA 2002-0879, January 2002

ii Jones, Thomas W., Lunsford, Charles B.; "Design and Development of a Real-Time Model Attitude Measurement System for Hypersonic Facilities", 43rd AIAA Aerospace Sciences Meeting and Exhibit, Reno, Nevada, AIAA 2005-1411, January 2005

${ }^{\text {iii }}$ Analog Devices; "Precision $\pm 1.7 \mathrm{~g}$ Single-/Dual-Axis $i$ MEMS ${ }^{\circledR}$ Accelerometer" Technical Data Sheet Revision March 2006, http://www.analog.com/UploadedFiles/Data_Sheets/178749895ADXL103_203_a.pdf Accessed May 2, 2006

iv Analog Devices; "Low-Cost_10 g Dual-Axis Accelerometer with Duty Cycle" Technical Data Sheet Revision May 2002, http://www.analog.com/UploadedFiles/Data Sheets/41294473836157ADXL210E 0.pdf Accessed May 2,2006

v Micol, J.R., "Langley Aerothermodynamic Facilities Complex: Enhancements and Testing Capabilities", AIAA Paper 98-0147, January 1998

${ }^{v i}$ Finley, Tom D.; “A Digital Pitch \& Roll Monitor", Proceedings of the $35^{\text {th }}$ International Instrumentation Symposium, ISA \#89-0023, May 1989
} 
${ }^{\text {vii }}$ Crawford, Bradley L., Finley, Tom D., "Improved Correction System for Vibration Sensitive Inertial Angle of Attack Measurement Devices", $38^{\text {th }}$ AIAA Aerospace Sciences Meeting \& Exhibit, Reno, Nevada, AIAA 20000415, January 2000 